\title{
Sulphadoxine-pyrimethamine plus azithromycin for the prevention of low birthweight in Papua New Guinea: a randomised controlled trial
}

Holger W Unger ${ }^{1,2}$, Maria Ome-Kaius ${ }^{2 \dagger}$, Regina A Wangnapi ${ }^{2 \dagger}$, Alexandra J Umbers ${ }^{1,2}$, Sarah Hanieh ${ }^{1}$, Connie SN Li Wai Suen ${ }^{3}$, Leanne J Robinson ${ }^{2,3}$, Anna Rosanas-Urgell ${ }^{2,4}$, Johanna Wapling ${ }^{2}$, Elvin Lufele ${ }^{2}$, Charles Kongs ${ }^{2}$, Paula Samol ${ }^{2}$, Desmond Sui ${ }^{2}$, Dupain Singirok ${ }^{2}$, Azucena Bardaji $1^{5}$, Louis Schofield ${ }^{3,6}$, Clara Menendez ${ }^{5}$, Inoni Betuela ${ }^{2}$, Peter Siba ${ }^{2}$, Ivo Mueller ${ }^{3,5,7}$ and Stephen J Rogerson ${ }^{1 *}$

\begin{abstract}
Background: Intermittent preventive treatment in pregnancy has not been evaluated outside of Africa. Low birthweight (LBW, $<2,500 \mathrm{~g}$ ) is common in Papua New Guinea (PNG) and contributing factors include malaria and reproductive tract infections.

Methods: From November 2009 to February 2013, we conducted a parallel group, randomised controlled trial in pregnant women ( $\leq 26$ gestational weeks) in PNG. Sulphadoxine-pyrimethamine $(1,500 / 75 \mathrm{mg})$ plus azithromycin (1 $\mathrm{g}$ twice daily for 2 days) (SPAZ) monthly from second trimester (intervention) was compared against sulphadoxinepyrimethamine and chloroquine (450 to $600 \mathrm{mg}$, daily for three days) (SPCQ) given once, followed by SPCQ placebo (control). Women were assigned to treatment (1:1) using a randomisation sequence with block sizes of 32. Participants were blinded to assignments. The primary outcome was LBW. Analysis was by intention-to-treat.
\end{abstract}

Results: Of 2,793 women randomised, 2,021 (72.4\%) were included in the primary outcome analysis (SPCQ: 1,008; SPAZ: 1,013). The prevalence of LBW was 15.1\% (305/2,021). SPAZ reduced LBW (risk ratio [RR]: 0.74, 95\% Cl: 0.60-0.91, $P=0.005$; absolute risk reduction (ARR): $4.5 \%, 95 \% \mathrm{Cl}: 1.4-7.6$; number needed to treat: 22$)$, and preterm delivery $(0.62$, 95\% Cl: 0.43-0.89, $P=0.010)$, and increased mean birthweight $(41.9 \mathrm{~g}, 95 \% \mathrm{Cl}: 0.2-83.6, P=0.049)$. SPAZ reduced maternal parasitaemia (RR: $0.57,95 \% \mathrm{Cl}: 0.35-0.95, P=0.029)$ and active placental malaria $(0.68,95 \% \mathrm{Cl}: 0.47-0.98$, $P=0.037)$, and reduced carriage of gonorrhoea $(0.66,95 \% \mathrm{Cl}: 0.44-0.99, P=0.041)$ at second visit. There were no treatment-related serious adverse events (SAEs), and the number of SAEs (intervention 13.1\% [181/1,378], control $12.7 \%[174 / 1,374], P=0.712$ ) and AEs (intervention 10.5\% [144/1,378], control 10.8\% [149/1,374], $P=0.737$ ) was similar. A major limitation of the study was the high loss to follow-up for birthweight.

Conclusions: SPAZ was efficacious and safe in reducing LBW, possibly acting through multiple mechanisms including the effect on malaria and on sexually transmitted infections. The efficacy of SPAZ in the presence of resistant parasites and the contribution of AZ to bacterial antibiotic resistance require further study. The ability of SPAZ to improve pregnancy outcomes warrants further evaluation.

Trial registration: ClinicalTrials.gov NCT01136850 (06 April 2010).

Keywords: Intermittent preventive treatment, Malaria, Pregnancy, Preterm delivery, Sexually transmitted infections

\footnotetext{
* Correspondence: sroger@unimelb.edu.au

${ }^{\dagger}$ Equal contributors

'Department of Medicine (Royal Melbourne Hospital), The University of Melbourne, Post Office Royal Melbourne Hospital, Parkville, Victoria 3050, Australia

Full list of author information is available at the end of the article
}

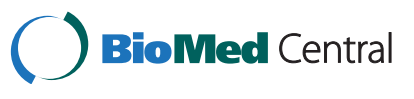

(c) 2015 Unger et al.; licensee BioMed Central. This is an Open Access article distributed under the terms of the Creative Commons Attribution License (http://creativecommons.org/licenses/by/4.0), which permits unrestricted use, distribution, and reproduction in any medium, provided the original work is properly credited. The Creative Commons Public Domain Dedication waiver (http://creativecommons.org/publicdomain/zero/1.0/) applies to the data made available in this article unless otherwise stated. 


\section{Background}

Infants born with low birthweight (LBW, $<2,500 \mathrm{~g}$ ) due to pre-term delivery (PTD, $<37$ weeks) and/or fetal growth restriction are at increased risk of morbidity and mortality [1,2]. Malaria in pregnancy is an important cause of fetal growth restriction, PTD, and adverse birth outcomes (miscarriage, stillbirth) [3], primarily due to inflammatory processes secondary to sequestration of Plasmodium falciparum (P.f.)-infected red blood cells in the placenta [4]. Many women with placental malaria are asymptomatic and such an infection can be undetectable by peripheral blood examination [2]. In areas of moderateto-high endemicity, primigravidae are most at risk [5]. The burden of malaria and LBW is highest in lowincome countries and, worldwide, 125.2 million pregnant women were at risk of infection in 2007 [6].

Intermittent preventive treatment of malaria in pregnancy (IPTp), namely the periodic administration of a curative dose of an antimalarial, provides intermittent chemoprophylaxis and clears asymptomatic infections. The World Health Organisation recommends monthly courses of sulphadoxine-pyrimethamine (SP) from second trimester in areas of Africa with moderate-to-high malaria transmission [7]. Although IPTp-SP remains generally efficacious, increasing drug resistance has generated a need for new IPTp candidates [8,9]. Azithromycin (AZ) is an azalide antibiotic with favourable antimalarial properties and a good safety profile in all trimesters of pregnancy [10]. AZ combined with SP (SPAZ) reduced PTD and malaria at delivery in one study in Malawi $[11,12]$. Further, AZ has the potential to simultaneously clear sexually transmitted infections (STIs) and reproductive tract infections that are known to increase the risk of PTD and adverse pregnancy outcomes [13]. The antimalarial effect of AZ has been associated with blood levels at $96 \mathrm{~h}$, and doses of 4 to $4.5 \mathrm{~g}$ over 2 to 3 days appear to be required to obtain appropriate levels $[10,14,15]$.

To date, IPTp has been studied only in Africa, where P.f. predominates, and IPTp use outside of Africa is currently not endorsed by the World Health Organisation [7]. However, many pregnant women elsewhere are at risk of malaria in areas, such as Papua New Guinea (PNG), where both P.f. and P. vivax (P.v.) are endemic $[3,16]$. P.v. also causes adverse pregnancy outcomes, through less well understood mechanisms [17].

We evaluated the efficacy and safety of IPTp with SPAZ to prevent LBW and to reduce the prevalence of malaria and anaemia at delivery in PNG.

\section{Methods}

Ethics

Ethical approval for the study protocol (Additional file 1) was obtained from the PNG Institute of Medical Research (PNGIMR) Institutional Review Board, the PNG Medical
Research Advisory Council, and the Melbourne Health Human Research Ethics Committee. The trial was registered with the United States National Institutes of Health Clinical Trials Registry (NCT01136850, registered 06 April 2010) and has been reported according to CONSORT guidelines (Additional file 2). Because there is currently insufficient evidence to support a general recommendation for the use of IPTp-SP outside Africa [7], and because SP alone is often ineffective against P.v. [18], which causes around $40 \%$ of malaria infections in PNG, we compared SPAZ-IPTp to a single course of $\mathrm{SP}$ and chloroquine (CQ) to eliminate infection. The study was conducted in accordance with Good Clinical Practice guidelines (ICH GCP E6). External monitoring was provided by one independent monitor and the Data Safety Monitoring Board (DSMB). All participants provided informed written consent. The legal age of consent for women in PNG is 16 years. The trial was registered in April 2010, after 336 women had joined the study, as a result of miscommunication between the principal investigator and the on-site clinical team.

\section{Interventions}

The trial had two treatment arms with a 1:1 allocation ratio. In the intervention arm, women received three courses of SP (3 tablets [500/25 mg] given once, Micro Labs Ltd., India) and AZ (2 tablets [500 mg] twice daily for 2 days, Pfizer, USA), at minimum intervals of 4 weeks. Women assigned to the control arm received one course of SP (3 tablets, $500 / 25 \mathrm{mg}$ ) and CQ (3 or 4 tablets [150 mg], daily for 3 days, Medopharm, India) at enrolment, followed by monthly courses of placebo equivalent (J. Bonal S.A., Spain). Women who did not already own an insecticide-treated net were given one at enrolment if available; local stock-outs meant that $8 \%$ of women did not own, or receive, bed nets.

Intake of SP and the first of four doses of AZ (1 g; intervention arm), and intake of SP (or placebo equivalent) and the first of three doses of CQ (or placebo equivalent; control arm) was supervised at an antenatal clinic. Drugs were administered with dry biscuits, and women were observed for a minimum of 30 minutes following ingestion of study medications. Treatment was rescheduled for first trimester pregnancies and women with a positive malaria rapid diagnostic test. Adherence to the remaining three doses of $\mathrm{AZ}$ or two doses of $\mathrm{CQ}$ (or CQ placebo equivalent) for first and second courses was assessed retrospectively at second and third study visits, respectively, through help of a questionnaire. Drug levels were not taken.

\section{Objectives and outcome measures}

The primary objective was to compare efficacy of IPTp with SPAZ with a single treatment course of SPCQ to prevent LBW. 
The primary outcome measure was the proportion of live born, singleton infants without congenital malformations with LBW. Secondary outcome measures included mean birthweight, the proportion of women with malaria and anaemia at delivery, and the proportion of women who delivered a preterm infant. Safety outcomes included the number of adverse events (AEs) and, specifically, the number of maternal deaths, stillbirths, neonatal deaths, and infants with congenital abnormalities.

\section{Trial design, setting, and participants}

We conducted a single-blinded block-randomised controlled trial.

The control arm was adapted from the policy for prevention of malaria in pregnancy in PNG when the trial was designed, which involved clearance of infection with a single dose of SP plus CQ for 3 days at first antenatal visit, followed by weekly doses of CQ until delivery (PNG standard of care) [16]. Because P.f. is highly resistant to CQ in PNG $[19,20]$, and compliance was poor, we instead endeavoured to provide all participants with insecticide-treated nets. To allow for participant blinding, women assigned to control treatment were provided placebo doses of SPCQ at subsequent study visits. A previous survey of molecular markers of SP resistance in children from the study area demonstrated absence of 'high' and 'super' resistant P.f. and a low prevalence of the dhps K540E mutation (20\%) $[18,21]$. The dose and regimen for AZ was selected based on previous pharmacokinetic and tolerability studies [10], and to give adequate drug levels at 96 hours to clear P.f. and P.v. [14,22].

Pregnant women were enrolled between 23 November 2009 and 15 August 2012 at nine antenatal clinics in the Madang and Sumkar districts of Madang Province, PNG (Additional file 3). Pregnancy outcome follow-up was concluded on 28 February 2013. Participating health facilities with labour wards included Modilon General Hospital, Yagaum Hospital, and the health centres in Alexishafen and Mugil.

In a 2006 survey at one of the participating health centres, prevalence at first antenatal visit of P.f. and P.v. was $30.3 \%$ and $8.1 \%$ (by light microscopy), respectively, and the prevalence of LBW amongst women not using insecticide-treated nets was $17 \%$ (unpublished data). The study area experiences year-round malaria transmission and was considered hyper-endemic at the time the trial was designed [23]. Chlamydia trachomatis, Neisseria gonorrhoeae, and syphilis are thought to be common in pregnant women in PNG [24]. Antenatal HIV prevalence at the provincial hospital (Modilon General Hospital) was 1.1\% (2009 - 2012, unpublished audit data).

\section{Screening, enrolment, and follow-up procedures}

Community campaigns were held to raise awareness of the trial. All pregnant women presenting for their first antenatal visit at participating health centres were invited to attend group information sessions and were screened. Interested women were excluded if they had i) gestation $>26$ weeks by abdominal palpation, ii) haemoglobin $<6 \mathrm{~g} / \mathrm{dL}$ and symptomatic as a result of anaemia, iii) previous serious adverse reaction to study medications, iv) permanent disability and chronic medical conditions, v) known multiple pregnancy, vi) unavailable for follow-up, or vii) age $<16$ years. We collected detailed information on reasons for non-inclusion at screening for a subset of antenatal recruitment clinic sessions $(n=279)$, during which $30.8 \%(860 / 2,793)$ of all women randomised to treatment were enrolled. Due to logistic constraints, we were unable to gather demographic and clinical background data on women who were screened but not enrolled. Written informed consent was obtained, a focussed clinical examination performed, and socio-demographic and clinical data collected using standardised case report forms. A venous blood sample was taken and peripheral blood smears were prepared. Women with malaria symptoms and/or haemoglobin $<9 \mathrm{~g} / \mathrm{dL}$ (HemoCue Ltd, Angelholm, Sweden; accuracy of $0.1 \mathrm{~g} / \mathrm{dL}$ ) were tested using a malaria rapid diagnostic test (CareStart ${ }^{\mathrm{TM}}$ P.f/Pan combo, AccessBio, USA). Anaemia and malaria were treated with iron/ folate supplements and albendazole, and quinine (in first trimester, $300 \mathrm{mg}, 2$ tablets orally 3 times daily for 7 days) or artemether-lumefantrine (in second and third trimesters, 20/120 mg, 4 tablets 6 times over 3 days), as per national protocol [25]. Women treated for malaria had their study medication administration rescheduled 2 weeks later. Women were screened for syphilis (Syphicheck-WB, QualPro, India) and treated with 2.4 MU of benzathine penicillin $\mathrm{G}$ if found positive.

We were able to offer a sub-set of participants an obstetric ultrasound (Logiqbook XP, General Electric Medical Systems, UK) within a week of enrolment; technical problems with our ultrasound machine precluded scanning for the entire trial period. Fetal biometry alone was used to estimate gestational age (GA), as the majority of women were unable to report their last menstrual period and/or menstrual cycle characteristics, and Ballard scores correlated poorly with GA in our cohort. For women presenting early, GA was estimated as per British Medical Ultrasound Guidelines [26]. Women who had their first scan after mid-second trimester had their GA estimated according to Hadlock et al. [27]. The earliest scan available for each woman was selected to estimate GA at delivery. At subsequent scheduled study visits, peripheral blood smears and samples were collected, and routine antenatal examination performed. 
At the second treatment visit, a self-collected vaginal swab was obtained for testing for $C$. trachomatis, $N$. gonorrhoeae, and Trichomonas vaginalis for a subset of women. Once available, participants were notified of results and referred for treatment.

Participants were followed-up until delivery, and birthweights measured by study nurses to the nearest $10 \mathrm{~g}$ using digital infant scales (Cupid 1, Charder Medical, Taiwan). Time elapsed between birth and birthweight measurements was documented, and newborns were checked for congenital abnormalities. Deliveries $<22$ gestational weeks were categorised as miscarriages. Maternal haemoglobin was measured, peripheral blood placental impression and cord blood smears prepared, and placental biopsies were taken. Women were invited to re-attend at 4 to 6 weeks postpartum with their baby. A team of community liaison officers, reporters, and nurses was dedicated to the follow-up of women who did not present for delivery at a participating health centre within one month of the estimated delivery date in order to establish pregnancy outcome.

\section{Adverse events monitoring and reporting}

Case report forms were completed for maternal and neonatal AEs detected at scheduled antenatal and postpartum visits, at delivery, and during non-scheduled visits. The study clinician on-call was alerted by the nursing team upon detection of a possible serious adverse event (SAE), whereby cases were clinically evaluated and reported shortly thereafter, but allowing for a maximum time frame of 24 hours for reporting of cases detected at distal study sites. AEs were considered SAEs if fulfilling one of the following criteria: the event resulted in death, was a congenital abnormality, resulted in hospitalisation or prolongation of existing hospitalisation, was life-threatening, resulted in persistent/ significant disability, or was deemed serious for other medically significant reasons by the study physicians. SAE reports were completed for mothers who experienced miscarriages or stillbirths. Analysis of all AEs was by actual drugs received (hence adjusted for crossover) and included all women who received trial medications $(\mathrm{n}=2,752)$. Drug-related AEs represented drug side effects. Reports of side effects at each treatment course (including placebo administration in the control arm) were considered as separate AEs.

A detailed report of each SAE was sent immediately to the DSMB, and the Malaria in Pregnancy Consortium and Pfizer drug safety groups. Assessments of the relationship between AEs and study medication were undertaken by the investigators and reports forwarded to the DSMB and an independent clinician for scrutinisation.

\section{Laboratory evaluations}

Labelled blood and impression smears were air-dried and stained with $4 \%$ Giemsa for 30 minutes. Thick smears were used to count the number of asexual parasites per 200 leukocytes (or per 500 if $<10$ parasites/200 leukocytes), assuming 8,000 leukocytes/ $\mu \mathrm{L}$ of blood; slides were declared negative if no parasite was seen in 200 oilimmersion fields. Two microscopists read each slide, and third reads were performed to resolve discrepant results. When species discrepancies remained after third reads, qPCR was performed on maternal venous blood, and these results were considered definitive [28]. A sample of $10 \mathrm{~mL}$ of venous blood was collected from each participant at both enrolment and delivery into Lithium Heparin vacutainers (BD, USA), and plasma was separated and stored at $-80^{\circ} \mathrm{C}$ until further analyses. Light microscopy and qPCR were undertaken at the PNGIMR. Vaginal swabs were stored, extracted, and analysed by qPCR for presence of beta globin (positive control), C. trachomatis, N. gonorrhoeae, and T. vaginalis as described elsewhere [29].

A placental biopsy $(2.5 \times 2.5 \times 1 \mathrm{~cm})$ was collected and included the thickness of the placenta from the maternal to the fetal side without reaching the fetal membrane. Biopsies were stored at room temperature in $10 \%$ neutral buffered formalin and trimmed to fit histology cassettes. Cassettes were transported to Melbourne, Australia, where they were wax embedded. Histological sections were stained with Giemsa and coverslipped. Slides were returned to the PNGIMR for analysis. A subset of 423 placental biopsies were read at CRESIB, Barcelona, Spain, by Prof J Ordi, who also provided quality control on slide reading. Placental malaria was staged according to the presence/absence of three histological features: infected erythrocytes, hemozoin in monocytes/macrophages, and hemozoin in fibrin deposits [30,31]. Placental malaria was classified as active (parasites detected) or past infection (malaria pigment without parasites) $[30,31]$.

\section{Randomisation and masking}

Following enrolment, women were randomly allocated to SPCQ/placebo or SPAZ using a randomisation list prepared by an independent statistician in Stata 10.0 (StataCorp, USA). Each treatment (SPCQ/placebo or SPAZ) was randomly assigned to four different treatment codes, resulting in a total of eight treatment codes (letters $\mathrm{A}-\mathrm{H})$. A block randomisation procedure was subsequently used, with blocks of 32 , each containing four women assigned to each treatment code. Study drugs were packaged and labelled at the PNGIMR by staff not involved in the trial, and allocation codes were kept in a locked file cabinet offsite. Women were randomised to treatment codes using pre-made, opaque, consecutively numbered envelopes. The treatment code was revealed after completion of enrolment and immediately 
prior to treatment. Taste and colour of placebo and active medication differed at times; enrolled women were not told of their allocation, but it was impossible to blind clinical staff directly involved with drug administration. All other personnel (laboratory and administrative staff, data entry clerks) were blinded to the assignments. The allocation code was broken at completion of laboratory analyses and upon finalisation of data collection, entry, and cleaning. The statistical analysis was performed after the database was locked, but was not blind to treatment allocation.

\section{Sample size calculations}

The sample size calculation was based on the assumption that SPAZ lowers the proportion of LBW babies by $30 \%$ compared to a single course of SP and CQ when given in conjunction with insecticide-treated nets (12.0\% to $8.4 \%$ ). Taking into consideration $20 \%$ loss to follow-up, a power of $80 \%$, and $95 \%$ confidence, 1,396 women per arm were needed to demonstrate efficacy (Additional file 1).

\section{Statistical analysis}

Data was double-entered into case report form-specific databases (FoxPro 9.0, Microsoft, USA). Individual databases were merged and data analysed using Stata 12.0 (StataCorp, USA). Both intention-to-treat (ITT) and perprotocol (PP) analyses were carried out for the primary outcome (LBW), birthweight, malaria, and anaemia at delivery. ITT analysis included all women randomised to treatment, except those that were retrospectively excluded because of incomplete consent forms. Women who experienced unintentional crossover were assessed by original assigned groups. PP analysis only included women who received two or three treatments without crossover. Safety analyses were performed for all women who received treatment, including those with incomplete consent forms, and were done according to actual treatment received at enrolment (adjusted for crossover). Women with incomplete consent forms (lack of appropriate signature, date, and/or witness if illiterate) had been screened, had received counselling, had provided written informed consent, and were randomised to treatment. As per the trial monitor's recommendations, these women were retrospectively excluded from all trial analyses bar those pertaining to drug safety.

Only birthweights from singleton pregnancies of live births $\geq 22$ weeks' gestation with no congenital abnormality and measured within 7 days of delivery were included in birthweight analyses [32].

To assess the distribution characteristics of continuous variables, data were graphed as a kernel density plot including a normal density and the Shapiro-Wilk test was performed. Linearity of continuous data was assessed through visual inspection of scatter plots. Univariate comparisons of variables were subsequently performed using the $x^{2}$ test or Fisher's exact test for categorical data, the Student's $t$-test or ANOVA for parametric data, and Mann Whitney-U or Kruskal-Wallis tests for nonparametric data. Unadjusted risk ratios (RR) were calculated for primary and secondary categorical outcomes including LBW, and difference in means were calculated for continuous parametric data including birthweight and haemoglobin. $P<0.05$ was considered significant.

All factors with a tendency for association with LBW when analysed univariately (defined as $P<0.1$ ) were included in a multivariable Poisson regression model with robust error variance as a starting model for a backward stepwise elimination model selection procedure [33]. A multivariable linear regression was used to calculate an adjusted difference in mean birthweight. Multivariate analyses for LBW/birthweight were performed in order to validate the effect of drug treatment on the primary outcome observed in crude analysis. For women with known pregnancy outcome but missing birthweight, multiple imputation was performed to derive estimated birthweights by drawing 20 imputed data sets from a Bayesian posterior predictive distribution of the missing data [34].

A priori tests for interaction (defined as $P<0.15$ of interaction term) between the intervention and gravidity (categorised as primigravida, multigravida), bed net use prior to enrolment (categorised as non-user, and user of a bed net [untreated and treated combined]), maternal height (categorised as low $[<150 \mathrm{~cm}]$ and normal height $[\geq 150 \mathrm{~cm}]$ ) and maternal ethnic grouping (categorised as maternal highlander parentage and non-highlander parentage) were performed on the final multivariable model for LBW using the Wald test. Stratified analyses for the primary outcome using the same variables were undertaken.

Lastly, we estimated the population-attributable fraction of LBW due to malaria using established methodology [1]. This calculation was based on the assumption that LBW can only be attributed to malaria if there was evidence of placental malaria (past or active infection).

\section{Role of funding source and ethical approval}

This trial was supported by the Malaria in Pregnancy Consortium (funded by the Bill \& Melinda Gates Foundation, 46099), and the Pregvax Consortium (European Union's Seventh Framework Programme FP7-2007HEALTH, PREGVAX 201588, and the Spanish Government EUROSALUD 208 Programme). Azithromycin was provided by Pfizer Inc. as part of an InvestigatorInitiated Research grant (WS394663). Funding sources did not have any involvement in study design, collection, analysis, and interpretation of data, and compilation and submission of this report. 


\section{Results}

Of women screened at antenatal clinics, 2,793 were enrolled between November 2009 and August 2012. The trial flowchart is outlined in Figure 1: 18 women were excluded due to incomplete consent forms, leaving 2,775 women (99.4\%) in the ITT cohort at baseline. Their demographic and clinical characteristics were similar across treatment arms and are presented in Table 1. Overall, $50.2 \%(1,390 / 2,770)$ of participants were primigravida, $62.2 \%(1,720 / 2,764)$ resided in rural areas, $81.4 \%$ $(2,157 / 2,650)$ were anaemic $(\mathrm{Hb}<11 \mathrm{~g} / \mathrm{dL})$, and $7.4 \%$ $(204 / 2,766)$ had microscopy-detected malaria parasitaemia. The principal reason for exclusion at screening was advanced GA (Figure 2).

Of women in the ITT cohort $81.0 \%(2,247 / 2,775)$ had birth outcome information collected (follow-up completed in February 2013), and $72.8 \%(2,021 / 2,775)$ had birthweights of congenitally normal live singletons measured within 7 days of delivery and were therefore eligible for inclusion in the primary outcome analysis. For 1,013 women who were randomised to SPAZ and included in the ITT birthweight analysis, the mean number of doses of SPAZ was $2.79 \pm 0.5$ (median 3, range 0-4). Women who were excluded from the ITT birthweight analysis $(\mathrm{n}=754)$ were more commonly malaria infected at baseline, had lower literacy, more commonly resided in rural areas, and had lower fundal heights compared to those included (Additional file 4), but had similar baseline characteristics when assessed according to treatment arm (Additional file 4). There was no significant difference in the proportion of women excluded from ITT birthweight analyses by trial arm (SPCQ 27.1\% [374/1,382] vs. SPAZ 27.3\% [380/1,393], $P=0.898$; Figure 1; Additional file 4). Amongst exclusions, 62.9\% (474/754) of women were lost for pregnancy outcome follow-up: their proportion did not differ between treatment arms (SPCQ 16.8\% [232/ $1,382]$ vs. SPAZ $17.4 \%[242 / 1,393], P=0.682)$.

The overall prevalence of LBW was $15.1 \%(305 / 2,021)$ and the mean birthweight was $2,943 \pm 479$ g. Amongst women who had pregnancy dating by ultrasound, $8.6 \%$ $(113 / 1,320)$ delivered preterm. Overall, $3.1 \%(63 / 2,045)$, $18.8 \%(278 / 1,472)$, and $7.4 \%(109 / 1,472)$ of women had peripheral parasitaemia, placenta malaria (past and active), and active placental malaria, respectively, and $74.4 \%$ $(1,389 / 1,868)$ were anaemic $(\mathrm{Hb}<11 \mathrm{~g} / \mathrm{dL})$ at delivery.

Compared to controls, women who received SPAZ had a lower risk of LBW (RR: 0.74, 95\% CI: 0.60-0.91, $P=0.005$ ), translating into an absolute risk reduction (ARR) of $4.5 \%$ (95\% CI: 1.4-7.6) and a number needed to treat of 22 (Table 2). Using imputed birthweights for 152 women with pregnancy outcome but missing birthweight, the RR for LBW with SPAZ was 0.74 (95\% CI: $0.60-0.92, P=0.007$ ). When adjusted for factors associated with LBW on univariate analysis, such as infant gender, gravidity, bed net use, maternal height, and maternal ethnic origin (Additional file 4), a similar RR (95\% CI) was obtained: 0.72 (0.59-0.89; $P=0.002$; Table 2 ). PP analyses yielded comparable results (Table 2).

Mean birthweight was $41.9 \mathrm{~g}$ higher (95\% CI: 0.2-83.6; $P=0.049)$ in the intervention arm, and when adjusted for potential confounders a similar result was obtained (52.4, 95\% CI: 13.2-91.6, $P=0.009$; Table 2). The observed difference in mean birthweight was largely explained by lower birthweights in the control compared to intervention in first quartile of the overall birthweight distribution (Mann Whitney-U test, $P=0.001$; Figure 3). In a subset of women who had ultrasound pregnancy-dating, a reduction in PTD in the intervention arm was noted (RR: 0.62, 95\% CI: $0.43-0.89, P=0.010$; ARR: $4.0 \%, 95 \%$ CI: $1.0-7.0$; Table 2).

We observed no statistically proven interaction on LBW between the intervention and gravidity $(P=0.728)$, bed net use prior to enrolment $(P=0.172)$, maternal height $(P=0.818)$, and maternal highlander parentage $(P=0.238)$. Stratification for the aforementioned variables suggested that women who benefitted most may have been those in their first pregnancy, of low height, of highlander parentage, and who reported bed net use at enrolment (Additional file 4).

Women receiving the intervention were at lower risk of peripheral (RR: $0.57,95 \%$ CI: $0.35-0.95 ; P=0.029$; ARR: 1.7\%, 95\% CI: 0.1-3.2) and placental blood parasitaemia (RR: 0.52 , 95\% CI: $0.28-0.97, P=0.034$; ARR: $1.6 \%$, 95\% CI: 0.1-3.2) as well as active placental infection (RR: 0.68, 95\% CI: 0.47-0.98, $P=0.037$; ARR: $2.9 \%$, 95\% CI: $0.2-5.5$; Table 3). There was no significant difference in mean $\mathrm{Hb}$ or in proportion of women with anaemia (Table 3). The population-attributable fraction of LBW due to malaria was estimated at $7.4 \%$ overall, and $0.9 \%$ and $12.1 \%$ in the intervention and control arms, respectively.

In self-collected vaginal swabs obtained from a subset of participants at second visit, carriage of $N$. gonorrhoeae was lower in women receiving SPAZ (SPCQ 8.2\% [55/674] vs. SPAZ 5.4\% [37/688], RR: 0.66, 95\% CI: 0.44-0.99, $P=0.041$ ), while carriage of $C$. trachomatis (SPCQ $4.5 \%$ [30/674] vs. SPAZ 3.8\% [26/688], RR: 0.85, 95\% CI: 0.51$1.42, P=0.532$ ) and $T$. vaginalis (SPCQ 21.8\% [147/674] vs. SPAZ 21.5\% [148/688], RR: 0.99, 95\% CI: 0.81-1.21, $P=0.894)$ did not differ significantly.

We detected 204 maternal and 151 neonatal SAEs, none of which were drug-related and there were no significant differences in numbers between treatment groups (Table 4). There were three maternal deaths (all due, or probably due, to postpartum haemorrhage). We did not observe significant differences in the proportion of maternal deaths, miscarriages, stillbirths, congenital abnormalities, and neonatal deaths between trial arms (Table 4). 


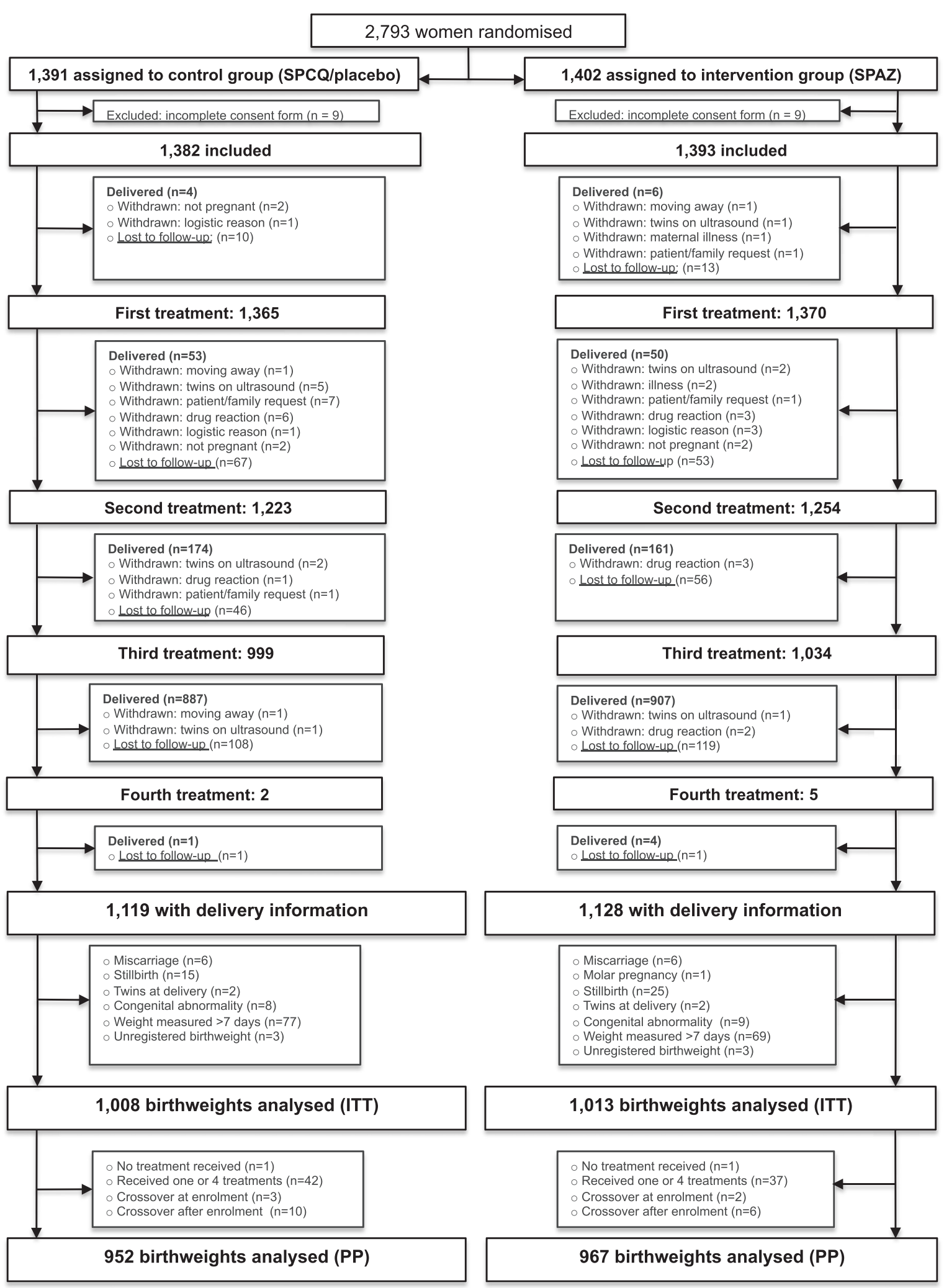

Figure 1 Trial profile. IPTp, Intermittent preventive treatment in pregnancy; SP, Sulphadoxine-pyrimethamine; CQ, Chloroquine; AZ, Azithromycin; ITT, Intention-to-treat analysis; PP, Per protocol analysis. 
Table 1 Baseline characteristics of study participants

\begin{tabular}{|c|c|c|c|c|}
\hline \multirow{3}{*}{$\begin{array}{l}\text { Characteristic } \\
\text { Age, years }\end{array}$} & \multicolumn{2}{|c|}{ Control (SPCQ and placebo) } & \multicolumn{2}{|c|}{ Intervention (SPAZ) } \\
\hline & \multicolumn{2}{|l|}{$(n=1,382)$} & \multicolumn{2}{|l|}{$(n=1,393)$} \\
\hline & 24.5 & {$[5.4]$} & 24.4 & {$[5.5]$} \\
\hline Height, $c m ; n=2,729$ & 154.3 & {$[6.0]$} & 154.1 & {$[5.8]$} \\
\hline Body mass index, $\mathrm{kg} / \mathrm{m}^{2} ; \mathrm{n}=2,721$ & 22.8 & {$[2.9]$} & 22.8 & {$[2.9]$} \\
\hline Mid-upper arm circumference, $\mathrm{cm} ; \mathrm{n}=2,714$ & 23.9 & {$[2.5]$} & 24.0 & {$[2.6]$} \\
\hline Fundal height, $\mathrm{cm} ; \mathrm{n}=2,771$ & 21.1 & {$[4.2]$} & 21.0 & {$[4.3]$} \\
\hline Haemoglobin $(\mathrm{Hb}), \mathrm{g} / \mathrm{dL} ; \mathrm{n}=2,650$ & 9.7 & {$[1.5]$} & 9.7 & {$[1.5]$} \\
\hline Anaemia, $\mathrm{Hb}<11 \mathrm{~g} / \mathrm{dL}$ & $1,058 / 1,313$ & $(80.6)$ & $1,099 / 1,337$ & $(82.2)$ \\
\hline Syphilis & $15 / 1,122$ & $(1.3)$ & $20 / 1,166$ & $(1.7)$ \\
\hline \multicolumn{5}{|l|}{ No. of previous pregnancies } \\
\hline 0 & 681 & (49.4) & 709 & $(51.0)$ \\
\hline 1 & 292 & $(21.2)$ & 279 & $(20.1)$ \\
\hline$\geq 2$ & 406 & (29.4) & 403 & $(29.0)$ \\
\hline Previous adverse pregnancy outcome & $103 / 1,375$ & $(7.5)$ & $119 / 1,389$ & $(8.6)$ \\
\hline \multicolumn{5}{|l|}{ Used bed net before enrolment ( 2 wks) } \\
\hline Not used & 322 & (23.4) & 347 & $(25.0)$ \\
\hline Used, without insecticide & 588 & $(42.7)$ & 561 & $(40.4)$ \\
\hline Used, insecticide treated & 466 & (33.9) & 481 & $(34.6)$ \\
\hline Given new bed net at enrolment & $873 / 1,375$ & $(63.5)$ & $849 / 1,385$ & $(61.3)$ \\
\hline Used antimalarials in this pregnancy & $146 / 1,340$ & $(10.9)$ & $161 / 1,366$ & $(11.8)$ \\
\hline \multicolumn{5}{|l|}{ Peripheral parasitaemia by microscopy } \\
\hline Any species ${ }^{a}$ & $100 / 1,376$ & $(7.3)$ & $104 / 1,390$ & $(7.5)$ \\
\hline Plasmodium falciparum & 87 & $(6.3)$ & 90 & $(6.5)$ \\
\hline Plasmodium vivax & 11 & $(0.8)$ & 12 & $(0.9)$ \\
\hline Smoker & $249 / 1,380$ & $(18.0)$ & 275/1,392 & $(19.8)$ \\
\hline Rural residence & $844 / 1,375$ & $(61.4)$ & $876 / 1,389$ & $(63.1)$ \\
\hline Literate & $1,223 / 1,376$ & $(88.9)$ & $1,248 / 1,391$ & $(89.7)$ \\
\hline Income-generating activity (woman) & $728 / 1,304$ & $(55.8)$ & $729 / 1,326$ & $(55.0)$ \\
\hline Income-generating activity (partner) & $939 / 1,358$ & $(69.2)$ & $947 / 1,378$ & $(68.7)$ \\
\hline Highlander parentage & $99 / 1,380$ & $(7.2)$ & 78/1,393 & $(5.6)$ \\
\hline
\end{tabular}

Data are mean [standard deviation], or number (\%).

ancludes two $P$. malariae in the control, and one $P$. malariae, one $P$. ovale, and one mixed $P$. falciparum and $P$. ovale infections in the intervention group.

There were a total of 293 drug-related AEs. The overall number of drug-related AEs did not differ between treatment groups, despite the fact that women in the control arm received placebo following their baseline treatment course $(P=0.737$; Table 4). Amongst women reporting drug-related AEs, common side effects included vomiting (5.7\%; 156/2,752), dizziness (3.2\%; 89/2,752), nausea (2.9\%; $80 / 2,752)$, itching $(1.0 \% ; 26 / 2,752)$, weakness $(1.0 \% ; 26 /$ $2,752)$, and abdominal pain $(0.6 \% ; 15 / 2,752)$. Women receiving control treatment more frequently experienced dizziness $(P=0.013)$ and abdominal pain was more commonly reported by women who had SPAZ $(P=0.021$; Table 4$)$.

Amongst women in the SPAZ arm, there was no difference in the proportion of women-infant pairs affected by at least one SAE (1 treatment: $14 / 117$ [12.0\%], 2 treatments: $23 / 223$ [10.3\%], 3+ treatments: 122/1,038 [11.8\%], $P$ [comparison across groups] $=0.821$ ), and there was no difference across groups when maternal SAEs (1 treatment: 13/117 [11.1\%], 2 treatments: 15/223 [6.7\%], 3+ treatments: 75/1,038 [7.2\%], $P$ [comparison across groups] $=0.285)$ and neonatal SAEs (1 treatment: 4/117 [3.4\%], 2 treatments: $12 / 223$ [5.4\%], 3+ treatments: $60 /$ 1,038 [5.8\%], $P$ [comparison across groups] $=0.567$ ) were evaluated separately. Similarly, the proportion of womeninfant pairs affected by at least one SAE did not differ significantly by number of treatment visits in the control arm (1 visit: 14/144 [9.7\%], 2 visits: 17/224 [7.6\%], 3+ visits: $116 / 1,006$ [11.5\%], $P$ [comparison across groups] $=0.208$ ). 


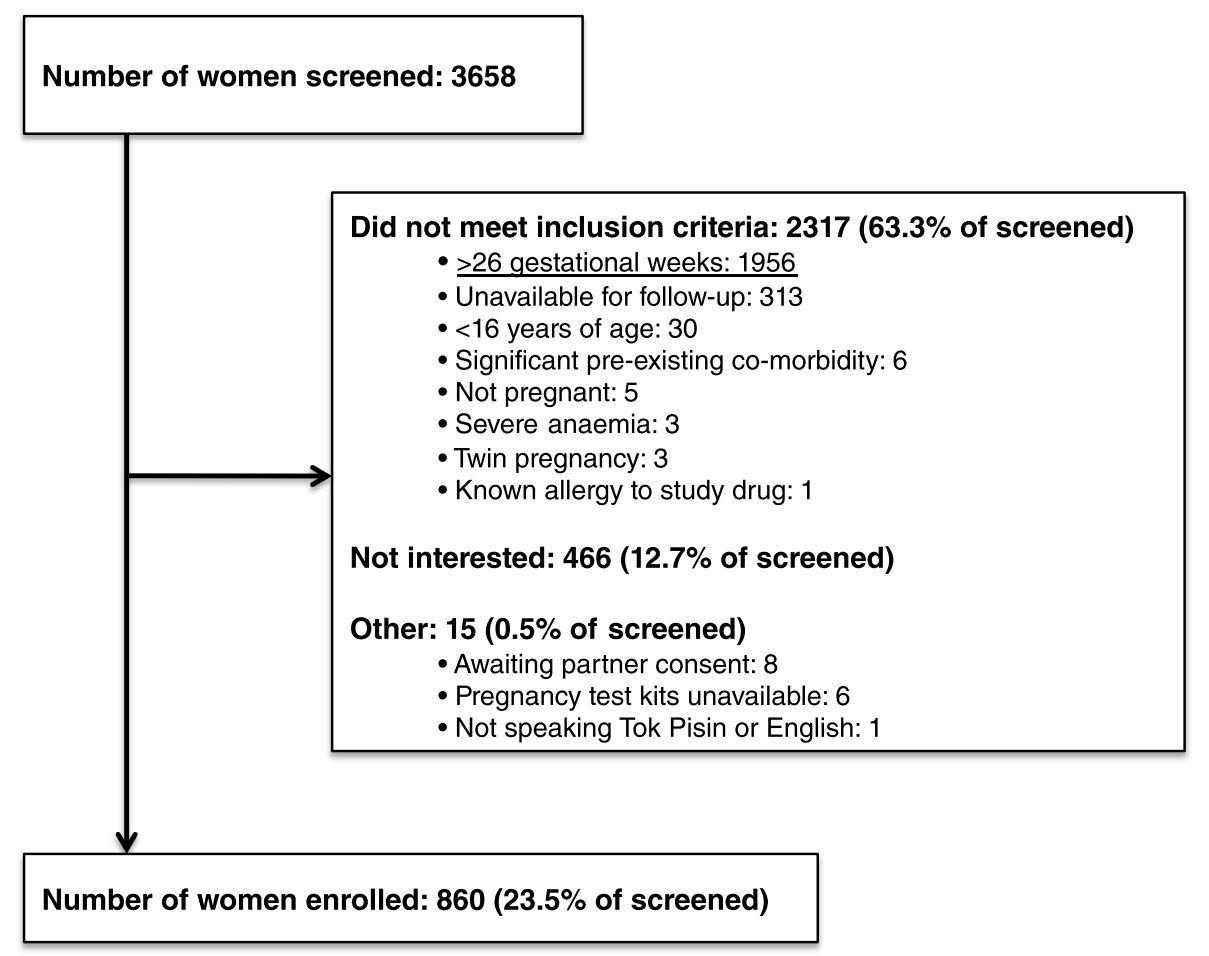

Figure 2 Detailed screening data for 279 clinic sessions (held at nine antenatal clinics) during which 860 of 2,793 trial participants $(30.8 \%)$ were recruited.

The proportion of women reporting at least one drugrelated $\mathrm{AE}$ increased with number of doses received, both for SPAZ (1 dose: 4/117 [3.4\%], 2 doses: 15/223 [6.7\%], 3+ doses: 116/1,038 [11.2\%], $P=0.007)$ and SPCQ/placebo (1 dose: 7/144 [4.9\%], 2 doses: 12/224 [5.4\%], 3+ doses 127/1,006 [10.6\%], $P<0.001)$. Only $1.9 \%$ $(47 / 2,466)$ and $1.1 \%(24 / 2,133)$ of women reported not taking the remaining doses of the treatment courses provided at enrolment and second study visit courses (AZ or $\mathrm{CQ} /$ placebo $\mathrm{CQ}$ ), respectively. There were no significant differences in adherence between treatment arms (enrolment treatment course: intervention 1.8\% [22/1,255] vs. control 2.1\% [25/1,211], $P=0.572$; second visit treatment course: intervention $1.2 \%[13 / 1,081]$ vs. control 1.1\% [11/1,052], $P=0.446)$.

\section{Discussion}

We found that IPTp with SPAZ significantly reduced the risk of LBW and PTD, and increased mean birthweight compared to a single treatment course of SPCQ. Both treatments were well tolerated.

To our knowledge, this is the first successful trial of IPTp outside of sub-Saharan Africa, or in an area where both P.f. and P.v. are endemic. The intervention reduced malaria infection at delivery, yet overall prevalence was substantially lower than previously reported [23]. IPTp with SPAZ appears to be beneficial against malaria in settings like PNG, although there were too few P.v. infections to evaluate the effect of the intervention on these. HIV infection is uncommon in Madang, precluding assessment of SPAZ in HIV-infected women.

Few participants reported problems with adherence to either study regime, despite the high dose of AZ. We only observed the first dose of each treatment course, and did not measure drug levels, hence non-adherence may be underestimated. The proportion of women reporting side effects in the intervention arm was small, despite the high dose of azithromycin $(4 \mathrm{~g})$ compared to other trials of IPTp testing AZ-based combinations $[11,15,35]$. This might be due to our split daily dosing, choice of partner drug for AZ, and provision of a dry biscuit prior to treatment at the antenatal clinic. Furthermore, the number of episodes during which women reported side-effects were similar between both groups, even though women randomised to the control arm had received placebo medication at follow-up visits.

The beneficial effect of SPAZ on LBW and placental malaria may, in part, be because of an imbalance in trial design as women in the intervention arm received more SP doses. Our control group was designed to match the current PNG protocol when the trial was designed. Although PNG policy now advocates three monthly doses of SP, a direct comparison with this regime is not possible: the impact of monthly SP on LBW in PNG is 
Table 2 LBW, preterm delivery, and mean birthweight, by treatment group

\begin{tabular}{|c|c|c|c|c|c|c|c|c|}
\hline \multirow[t]{2}{*}{ Outcome } & \multicolumn{3}{|l|}{ ITT analysis } & \multirow[b]{2}{*}{$P$} & \multicolumn{3}{|l|}{ PP analysis } & \multirow[b]{2}{*}{$P$} \\
\hline & $\begin{array}{l}\text { Control (SPCQ } \\
\text { and placebo) }\end{array}$ & $\begin{array}{l}\text { Intervention } \\
\text { (SPAZ) }\end{array}$ & $\begin{array}{l}\text { Risk ratio or } \Delta \\
\text { mean }(95 \% \mathrm{Cl})\end{array}$ & & $\begin{array}{l}\text { Control (SPCQ } \\
\text { and placebo) }\end{array}$ & Intervention (SPAZ) & $\begin{array}{l}\text { Risk ratio or } \Delta \\
\text { mean }(95 \% \mathrm{Cl})\end{array}$ & \\
\hline LBW (Birthweight $<2,500 \mathrm{~g}$ ), unadjusted & 175/1,008 (17.4) & $130 / 1,013(12.8)$ & $0.74(0.60-0.91)$ & 0.005 & $159 / 952(16.7)$ & 122/967 (12.6) & $0.76(0.61-0.94)$ & 0.011 \\
\hline LBW (Birthweight $<2,500$ g), adjusted ${ }^{a}$ & - & - & $0.72(0.59-0.89)$ & 0.002 & - & - & $0.72(0.58-0.89)$ & 0.003 \\
\hline Birthweight (g) & $2,921.6$ & $2,963.5$ & 41.9 & 0.049 & $2,928.7$ & $2,967.9$ & 39.2 & 0.068 \\
\hline unadjusted & {$[2,890.1-2,953.1]$} & {$[2,936.0-2,991.0]$} & $(0.2-83.6)$ & & {$[2,986.9-2,960.4]$} & {$[2,940.3-2,995.4]$} & $(-2.8-81.2)$ & \\
\hline Birthweight (g) & $2,916.6$ & $2,969.0$ & 52.4 & 0.009 & 2922.9 & 2974.1 & 51.2 & 0.011 \\
\hline adjusted $^{b}$ & {$[2,888.8-2944.3]$} & {$[2,941.4-2,996.6]$} & $(13.2-91.6)$ & & {$[2,895.0-2,950.9]$} & {$[2,946.4-3,001.8]$} & $(11.7-90.6)$ & \\
\hline $\begin{array}{l}\text { PTD (<37 GWs), n (\%), unadjusted, } \\
\text { best available dating ultrasound }\end{array}$ & 69/652 (10.6) & $44 / 668(6.6)$ & $0.62(0.43-0.89)$ & 0.010 & 63/622 (10.1) & 40/651 (6.1) & $0.61(0.41-0.89)$ & 0.009 \\
\hline
\end{tabular}

Data are number (\%) or mean [95\% Cl], unless stated otherwise; ITT, Intention-to-treat; PP, Per-protocol; GW, Gestational weeks; $\mathrm{Cl}$, Confidence interval. $P<0.05$ marked in bold.

${ }^{a}$ Adjusted for infant gender, gravidity, no. of treatment courses (ITT only), clinic location, season of delivery, bed net use, mid-upper arm circumference, height, maternal highlander heritage.

${ }^{\mathrm{b}}$ Adjusted for infant gender, gravidity, no. of treatment courses (ITT only), clinic location, mid-upper arm circumference, height, maternal highlander heritage. 


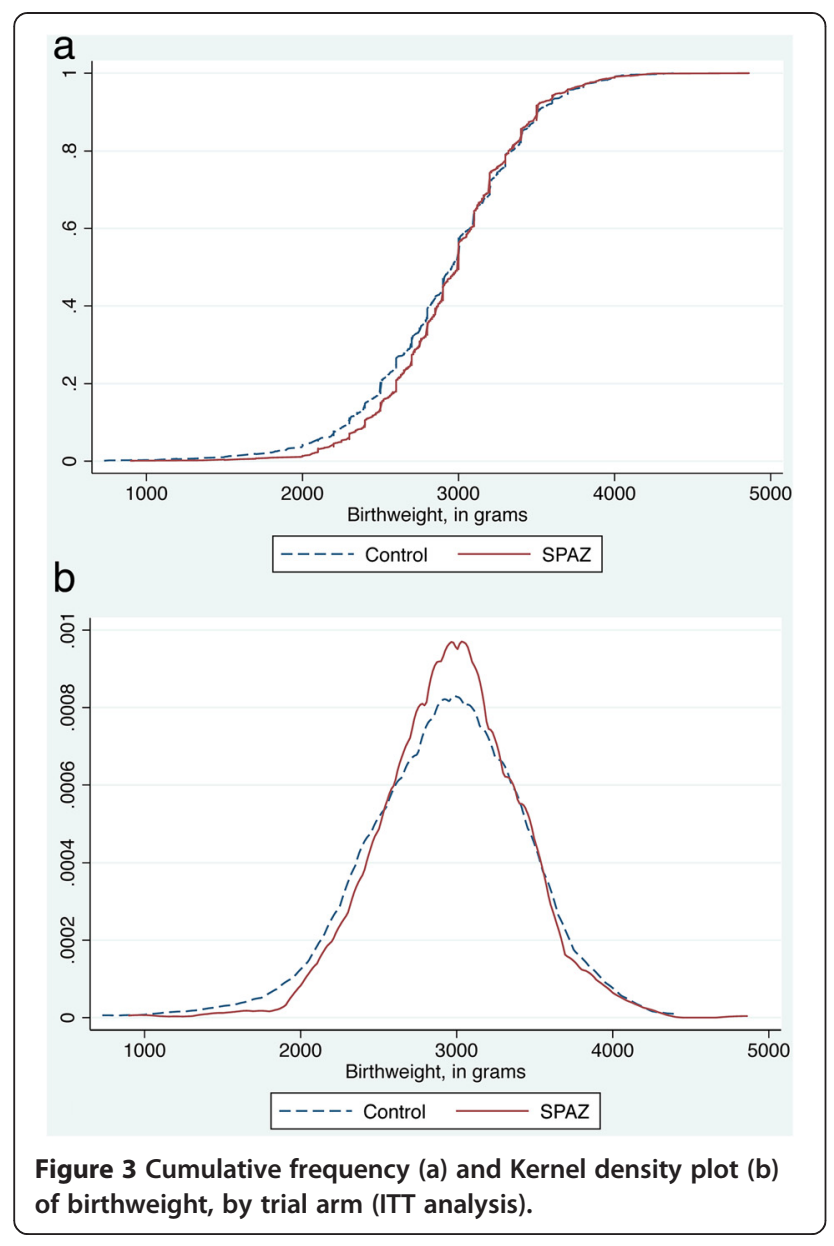

unknown. A recent meta-analysis demonstrated that three or more doses of SP are better than one or two for the prevention of malaria and LBW in African women [36]. If SPAZ were compared to monthly SP, the effect on LBW might be less striking. SP has not been shown to prevent PTD [37], which was significantly less frequent in women receiving SPAZ than SPCQ. The small number of women who received only one course of SPAZ or SPCQ and were followed up for the primary outcome $(n=75)$ precludes further analysis to quantify the benefit of adding AZ to SP. Interaction and stratified analyses did not indicate a difference in the efficacy of treatment by gravidity: the study was not powered and was not designed to convincingly demonstrate this.

The low overall prevalence of malaria (and low population attributable fraction of LBW due to malaria), as well as the reduction in PTD in the intervention arm, suggests that the effect of SPAZ on reducing LBW is mediated by other mechanisms in addition to its antimalarial effect. A greater impact on LBW might be observed in settings where malaria prevalence is higher than in this study, and women share other common risk factors. SPAZ may prevent LBW by clearing STIs and ascending reproductive tract infections [22], which are common in PNG [24]. Vaginal swabs were collected from a subset of 400 study participants at enrolment. Of these, $11 \%$ carried $C$. trachomatis, and $10 \%$ carried $N$. gonorrhoeae [29]. There were only modest differences in carriage of $C$. trachomatis and $N$. gonorrhoeae at second treatment visit between women receiving SPAZ and SPCQ. Carriage of $N$. gonorrhoeae was lower in women receiving SPAZ, while carriage of $C$. trachomatis did not differ significantly by treatment arm. This suggests that an impact on STI carriage explains only a part of the positive effect of SPAZ on LBW. AZ may also exert immunomodulatory effects favouring fetal growth [38].

Two previous studies of SPAZ-IPTp in rural Malawi gave conflicting results. In one, monthly SP and $1 \mathrm{~g}$ of $\mathrm{AZ}$ given twice reduced the risk of PTD, LBW, and malaria compared to two doses of SP, to a similar extent to our study $[11,12]$. In the other, addition of AZ $1 \mathrm{~g}$ twice to three doses of SP-IPTp did not significantly reduce PTD or malaria; LBW was not reported and mean birthweight was $40 \mathrm{~g}$ higher in the AZ arm [35]. Both studies used lower doses of AZ than our trial $(4 \mathrm{~g})$. Taken together, the studies suggest SPAZ may have beneficial effects on PTD, depending on the population characteristics.

One concern with use of SP is emergence of drug resistance that limits efficacy or could even exacerbate infection [39]. Parasites from this study have not been typed for drug resistance markers, but contemporaneous parasites from children and adults in the same locale have recently been analysed [18]. 'Highly resistant' patterns of molecular markers (quintuple mutations in the dhfr and dhps genes) and 'super resistant' parasites (also featuring dhfr 164 or dhps 581 mutations) [21] have not been detected, and the prevalence of dhps 540 mutations associated with drug failure in young children [21] was $<20 \%$. By contrast, P.v. is frequently resistant to SP [18], but was rare in this cohort.

Potential adverse consequences of AZ use may include selection for (probably temporary) increases in carriage of AZ-resistant organisms, especially Streptococcus pneumoniae [40], and a possible association between macrolide use in late pregnancy and increased risk of infantile hypertrophic pyloric stenosis [41]. Such potential effects require careful monitoring in future studies of $\mathrm{AZ}$ for indications such as IPTp.

There are several limitations to our study. First, birthweights eligible for inclusion in the primary outcome analysis were available for only $72 \%$ of women randomised to treatment. Reassuringly, loss to follow-up rates did not differ between control and intervention arm. Furthermore, there were no major differences in the background characteristics of women that were lost to follow-up when compared to those who were not (Additional file 4). However, women lost to follow-up were more likely to be young and malaria infected at enrolment. It 
Table 3 Malaria infection and anaemia at delivery, by treatment groups

\begin{tabular}{|c|c|c|c|c|c|c|c|c|c|c|c|c|}
\hline \multirow[t]{2}{*}{ Outcome } & \multicolumn{6}{|c|}{ ITT analysis } & \multicolumn{6}{|c|}{ PP analysis } \\
\hline & \multicolumn{2}{|c|}{$\begin{array}{l}\text { Control } \\
\text { (SPCQ and } \\
\text { placebo) }\end{array}$} & \multicolumn{2}{|c|}{$\begin{array}{l}\text { Intervention } \\
\text { (SPAZ) }\end{array}$} & \multirow[t]{2}{*}{$\begin{array}{l}\text { Risk ratio } \\
(95 \% \mathrm{Cl})\end{array}$} & \multirow[t]{2}{*}{$P$} & \multicolumn{2}{|c|}{$\begin{array}{l}\text { Control } \\
\text { (SPCQ and } \\
\text { placebo) }\end{array}$} & \multicolumn{2}{|c|}{$\begin{array}{l}\text { Intervention } \\
\text { (SPAZ) }\end{array}$} & \multirow[t]{2}{*}{$\begin{array}{l}\text { Risk ratio } \\
(95 \% \mathrm{Cl})\end{array}$} & $P$ \\
\hline \multicolumn{10}{|l|}{$\begin{array}{l}\text { Peripheral blood } \\
\text { (light microscopy) }\end{array}$} & & & \\
\hline All infections & $40 / 1,022$ & (3.9) & $23 / 1,023$ & $(2.3)$ & $0.57(0.35-0.95)$ & 0.029 & $39 / 962$ & $(4.1)$ & 22/972 & $(2.3)$ & $0.56(0.33-0.93)$ & 0.024 \\
\hline P. falciparum & 29 & $(2.8)$ & 18 & $(1.8)$ & $0.62(0.35-1.11)$ & 0.104 & 28 & $(2.9)$ & 17 & $(1.8)$ & $0.60(0.33-1.09)$ & 0.090 \\
\hline P. vivax & 11 & $(1.1)$ & 5 & $(0.5)$ & $0.45(0.16-1.30)$ & 0.142 & 11 & $(1.1)$ & 5 & $(0.5)$ & $0.45(0.16-1.29)$ & 0.127 \\
\hline \multicolumn{13}{|l|}{$\begin{array}{l}\text { Placental blood } \\
\text { (light microscopy) }\end{array}$} \\
\hline All infections & $29 / 847$ & (3.4) & $15 / 841$ & $(1.8)$ & $0.52(0.28-0.97)$ & 0.035 & $28 / 800$ & (3.5) & 14/803 & $(1.7)$ & $0.50(0.26-0.94)$ & 0.028 \\
\hline P. falciparum & 25 & (3.0) & 15 & $(1.8)$ & $0.60(0.32-1.14)$ & 0.115 & 24 & (3.0) & 14 & $(1.7)$ & $0.58(0.30-1.12)$ & 0.098 \\
\hline P. vivax & 1 & $(0.1)$ & 0 & $(0.0)$ & - & - & 1 & $(0.1)$ & 0 & $(0.0)$ & - & - \\
\hline Cord blood (light microscopy) ${ }^{a}$ & $4 / 744$ & $(0.5)$ & $3 / 744$ & $(0.4)$ & $0.75(0.17-3.34)$ & 1.000 & $4 / 695$ & $(0.6)$ & $3 / 716$ & $(0.4)$ & $0.73(0.16-3.24)$ & 0.681 \\
\hline \multicolumn{13}{|l|}{ Placental malaria (histology) } \\
\hline Active and past infection ${ }^{b}$ & $150 / 736$ & $(20.4)$ & $128 / 736$ & $(17.4)$ & $0.85(0.69-1.06)$ & 0.143 & 138/697 & $(19.8)$ & $122 / 702$ & $(17.4)$ & $0.88(0.71-1.10)$ & 0.245 \\
\hline Active infection $^{c}$ & $65 / 736$ & $(8.8)$ & $44 / 736$ & $(6.0)$ & $0.68(0.47-0.98)$ & 0.037 & $62 / 697$ & $(8.9)$ & $41 / 702$ & $(5.8)$ & $0.66(0.45-0.96)$ & 0.029 \\
\hline Anaemia $(\mathrm{Hb}<11 \mathrm{~g} / \mathrm{dL})$ & 689/935 & $(73.7)$ & 700/933 & $(75.0)$ & $1.02(0.97-1.07)$ & 0.508 & $647 / 883$ & $(73.3)$ & $663 / 889$ & $(74.6)$ & $1.02(0.96-1.08)$ & 0.532 \\
\hline Severe anaemia ( $\mathrm{Hb}<7 \mathrm{~g} / \mathrm{dL})$ & $44 / 935$ & $(4.7)$ & $37 / 933$ & $(4.0)$ & $0.84(0.55-1.29)$ & 0.432 & $39 / 883$ & $(4.4)$ & $34 / 889$ & $(3.8)$ & $0.87(0.55-1.36)$ & 0.531 \\
\hline
\end{tabular}

Data are number (\%). $P<0.05$ marked in bold.

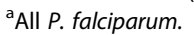

${ }^{b}$ Detection of parasites or malaria pigment.

'Detection of parasites.

is hence possible that this may have led to an underestimation of the effect of SPAZ on LBW, given these women are at higher risk of placental malaria [42], and may be at higher risk of carrying bacterial STIs [43], and so would benefit most from the intervention. Furthermore, the reasons for not presenting for delivery remain unknown for 474 women, which may include AEs. Second, the proportion of primigravidae was high (50.2\%), suggesting that there was selection bias, which could result in overestimating the effect SPAZ had on LBW. Third, only one quarter of women screened were ultimately enrolled and randomised, the principal reason for exclusion being presentation at advanced GA. This raises questions as to the representativeness of the study sample. Due to logistic reasons, we were unable to collect background demographic data for a substantial proportion of those women that were screened but not enrolled. It is possible that these women were more likely to be multigravid, and were more likely to be excluded because they tended to present at more advanced gestation. This may result in overestimation of the effect, although this could be less marked in circumstances of relatively low malaria prevalence. Not all women had ultrasound dating, and amongst those who did, many presented relatively late: use of later scans will underestimate GA in small-for-gestationalage babies and overestimate GA in macrosomic babies.
Reassuringly, there was a similar degree of reduction in PTD in the SPAZ arm amongst women who had early dating scans, although this was not statistically significant (due to a lack of power) (SPCQ 6.6\% [23/333] vs. SPAZ 3.9\% [13/327], RR: 0.58, 95\% CI: 0.30-1.12, $P=0.097)$. In addition, we did not measure in detail some potentially important confounders, including women's socioeconomic status. Lastly, due to funding constraints, we were unable to evaluate the impact of treatment on reducing chorioamnionitis, an important risk factor for PTD. Strengths of the study include the large sample size, random group allocation, the demonstration of benefit from IPTp in a setting outside Africa, and the impact of SPAZ on clinically-important endpoints of LBW and PTD.

The main reason for not meeting trial eligibility criteria was advanced GA, and there was a treatment-independent benefit of number of study visits in reducing LBW (Additional file 4). The more often, and earlier, women attend antenatal care, the more they will benefit from interventions that may reduce the risk of LBW other than IPTp, as well as maximise the benefit from IPTp [36]. It is therefore of utmost importance that access to, and early first attendance at, antenatal care is improved whilst interventions to improve birth outcomes are rolled out to prevent compromising the effectiveness of IPTp. Women excluded from the primary outcome analysis tended to be younger and were more likely to 
Table 4 Safety of trial interventions: adverse events

\begin{tabular}{|c|c|c|c|c|c|c|}
\hline \multirow{2}{*}{$\begin{array}{l}\text { Outcome } \\
\text { All adverse events }\end{array}$} & \multicolumn{2}{|c|}{$\begin{array}{l}\text { Control (SPCQ and placebo) } \\
n=1,374\end{array}$} & \multicolumn{2}{|c|}{$\begin{array}{l}\text { Intervention (SPAZ) } \\
\mathrm{n}=1,378\end{array}$} & \multirow{2}{*}{$\begin{array}{l}\text { Risk ratio }(95 \% \mathrm{Cl}) \\
0.96(0.85-1.07)\end{array}$} & \multirow{2}{*}{$\begin{array}{l}P \\
0.447\end{array}$} \\
\hline & 414 & $(30.1)$ & 397 & $(28.8)$ & & \\
\hline All serious adverse events (SAE) & 174 & $(12.7)$ & 181 & $(13.1)$ & $1.04(0.85-1.26)$ & 0.712 \\
\hline Maternal $^{\mathrm{A}}$ & 100 & $(7.3)$ & 104 & (7.5) & $1.04(0.80-1.35)$ & 0.788 \\
\hline No. admitted $^{\mathrm{b}}$ & 94 & $(6.8)$ & 90 & $(6.5)$ & $0.96(0.72-1.26)$ & 0.745 \\
\hline No. of mothers with 2 SAEs & 4 & $(0.3)$ & 1 & $(0.1)$ & $0.25(0.03-2.23)$ & 0.218 \\
\hline No. drug-related SAES & 0 & $(0.0)$ & 0 & $(0.0)$ & - & - \\
\hline \multicolumn{7}{|l|}{ Characteristics of maternal SAEs ${ }^{\mathrm{C}}$} \\
\hline Maternal death & 1 & $(0.1)$ & 2 & $(0.2)$ & $1.99(0.18-22.0)$ & $>0.999$ \\
\hline Spontaneous abortion & 4 & $(0.3)$ & 1 & $(0.1)$ & $0.24(0.03-2.23)$ & 0.218 \\
\hline Stillbirth & 15 & $(1.1)$ & 25 & $(1.8)$ & $1.66(0.88-3.14)$ & 0.113 \\
\hline Emergency caesarean section & 24 & $(1.8)$ & 24 & $(1.7)$ & $1.00(0.57-1.75)$ & 0.992 \\
\hline Hypertensive disorders of pregnancy & 11 & $(0.8)$ & 12 & $(0.9)$ & $1.09(0.48-2.46)$ & $>0.999$ \\
\hline Malaria & 6 & $(0.4)$ & 3 & $(0.2)$ & $0.50(0.12-1.99)$ & 0.314 \\
\hline Other infections & 10 & $(0.8)$ & 6 & $(0.4)$ & $0.60(0.22-1.64)$ & 0.330 \\
\hline Anaemia & 3 & $(0.2)$ & 2 & $(0.2)$ & $0.66(0.11-3.97)$ & 0.687 \\
\hline Placenta praevia & 2 & $(0.2)$ & 3 & $(0.2)$ & $1.50(0.25-8.94)$ & $>0.999$ \\
\hline Antepartum haemorrhage & 3 & $(0.2)$ & 4 & $(0.3)$ & $1.33(0.30-5.93)$ & $>0.999$ \\
\hline Preterm labour & 16 & $(1.2)$ & 6 & $(0.4)$ & $0.31(0.11-0.85)$ & 0.034 \\
\hline Preterm premature rupture of membranes & 13 & $(1.0)$ & 4 & $(0.3)$ & $0.31(0.10-0.94)$ & 0.030 \\
\hline Prolonged prelabour rupture of membranes & 2 & $(0.2)$ & 1 & $(0.1)$ & $0.50(0.05-5.49)$ & 0.624 \\
\hline Induction of labour (post-dates) & 0 & $(0.0)$ & 4 & $(0.3)$ & - & 0.125 \\
\hline Postpartum haemorrhage & 16 & $(1.2)$ & 23 & $(1.7)$ & $1.43(0.76-2.70)$ & 0.333 \\
\hline Other $^{d}$ & 3 & $(0.2)$ & 3 & $(0.2)$ & $1.00(0.20-4.93)$ & $>0.999$ \\
\hline Neonatal & 74 & $(5.4)$ & 77 & (5.6) & $1.04(0.76-1.42)$ & 0.816 \\
\hline No. admitted ${ }^{b}$ & 61 & $(4.4)$ & 67 & $(4.9)$ & $1.10(0.78-1.54)$ & 0.599 \\
\hline No. of babies with two SAEs & 0 & $(0.0)$ & 1 & $(0.0)$ & - & $>0.999$ \\
\hline No. drug-related SAES & 0 & $(0.0)$ & 0 & $(0.0)$ & - & - \\
\hline \multicolumn{7}{|l|}{ Characteristics of neonatal SAEs ${ }^{\mathrm{C}}$} \\
\hline Congenital abnormality ${ }^{\mathrm{e}}$ & 8 & $(0.6)$ & 10 & $(0.7)$ & $1.25(0.49-3.14)$ & 0.814 \\
\hline Neonatal death & 19 & $(1.4)$ & 11 & $(0.8)$ & $0.58(0.28-1.21)$ & 0.140 \\
\hline Prematurity & 15 & $(1.1)$ & 9 & $(0.7)$ & $0.60(0.26-1.36)$ & 0.227 \\
\hline Low birthweight & 20 & $(1.5)$ & 10 & $(0.7)$ & $0.50(0.23-1.06)$ & 0.069 \\
\hline Infection & 42 & $(3.1)$ & 37 & $(2.7)$ & $0.88(0.57-1.36)$ & 0.570 \\
\hline Birth asphyxia & 16 & $(1.2)$ & 18 & $(1.3)$ & $1.12(0.57-2.19)$ & 0.736 \\
\hline Meconium aspiration syndrome & 10 & $(0.7)$ & 14 & $(1.0)$ & $1.40(0.62-3.13)$ & 0.416 \\
\hline Cephalohaematoma & 3 & $(0.2)$ & 6 & $(0.4)$ & $1.99(0.50-7.96)$ & 0.507 \\
\hline Jaundice & 2 & $(0.2)$ & 3 & $(0.2)$ & $1.50(0.25-8.94)$ & $>0.999$ \\
\hline All non-serious adverse events (AEs) & 240 & $(17.5)$ & 216 & $(15.7)$ & $0.94(0.80-1.11)$ & 0.456 \\
\hline No. women with two AEs & 10 & $(0.7)$ & 13 & $(0.9)$ & $1.30(0.57-2.95)$ & 0.535 \\
\hline No. drug-related maternal $A E^{f}$ & 149 & $(10.8)$ & 144 & $(10.5)$ & $0.96(0.78-1.20)$ & 0.737 \\
\hline No. drug-related formal withdrawals ${ }^{9}$ & 7 & $(0.5)$ & 8 & $(0.6)$ & $1.14(0.41-3.13)$ & $>0.999$ \\
\hline No. women with two drug-related AEs & 3 & $(0.2)$ & 9 & $(0.7)$ & $2.99(0.81-11.03)$ & 0.145 \\
\hline
\end{tabular}


Table 4 Safety of trial interventions: adverse events (Continued)

\begin{tabular}{|c|c|c|c|c|c|c|}
\hline \multicolumn{7}{|c|}{ Characteristics of drug-related maternal AEs } \\
\hline Vomiting & 82 & $(6.0)$ & 74 & $(5.4)$ & $0.90(0.66-1.22)$ & 0.498 \\
\hline Dizziness & 56 & $(4.1)$ & 33 & $(2.4)$ & $0.59(0.39-0.90)$ & 0.013 \\
\hline Nausea & 37 & $(2.7)$ & 43 & $(3.1)$ & $1.16(0.75-1.79)$ & 0.544 \\
\hline Pruritus & 17 & $(1.2)$ & 9 & $(0.7)$ & $0.53(0.24-1.18)$ & 0.120 \\
\hline Weakness & 13 & $(1.0)$ & 13 & $(0.9)$ & $1.00(0.46-2.14)$ & 0.994 \\
\hline Abdominal pain & 3 & $(0.2)$ & 12 & $(0.9)$ & $4.32(1.23-15.13)$ & 0.021 \\
\hline Headache & 8 & $(0.6)$ & 5 & $(0.4)$ & $0.62(0.20-1.90)$ & 0.422 \\
\hline Diarrhoea & 1 & $(0.1)$ & 3 & $(0.2)$ & $2.99(0.31-28.7)$ & 0.625 \\
\hline Facial swelling (mild) & 3 & $(0.2)$ & 1 & $(0.1)$ & $0.33(0.04-3.19)$ & 0.374 \\
\hline Feeling hot & 3 & $(0.2)$ & 0 & $(0.0)$ & - & 0.124 \\
\hline Dyspepsia & 0 & $(0.0)$ & 4 & $(0.3)$ & - & 0.125 \\
\hline Loss of appetite & 0 & $(0.0)$ & 2 & $(0.2)$ & - & 0.500 \\
\hline Other & 5 & $(0.4)$ & 7 & $(0.1)$ & $1.40(0.44-4.39)$ & 0.774 \\
\hline
\end{tabular}

Data are $\mathrm{n}(\%)$. Five women with SAEs, and six with drug-related AEs (occurring after administration of the first, correct, treatment) had unintentional treatment crossover and were analysed as per original assignment. $P<0.05$ marked in bold.

anclude stillbirths and miscarriages.

${ }^{\mathrm{b}}$ Admission/prolongation of admission because of SAE.

'SAE reported because of one or more of the following.

dTrauma (2), vaginal haematoma (1), attempted suicide (1), gestational diabetes (1), hyperemesis gravidarum, possible appendicitis (1).

'Major: spina bifida (1), talipes equinovarus (5), cheilo- and palatoschisis [one with concomitant polydactyly] (2), prune belly syndrome (1), hypospadias (1), trisomy 21

(1), pulmonary atresia (1), multiple abnormalities of unknown cause (2), unilateral hand deformity (1) polydactyly (1), oligodactyly (1); minor: pectus carinatum (1).

f In the control group 19 women reported a reaction after taking placebo tablets.

${ }^{\mathrm{g}}$ All due to nausea/vomiting after taking the study medication.

be illiterate and reside in rural areas; such women might derive most benefit from interventions such as IPTp, especially when combined with early attendance at antenatal clinics [44].

\section{Conclusions}

Our findings suggest that IPTp with SPAZ reduces the risk of LBW in a setting of low-to-moderate malaria prevalence; it might have greater benefit in areas with higher malaria burden. Future research will evaluate the impact of SPAZ on pneumococcal antibiotic resistance, the latter being a potential barrier to implementation. Promising interventions to reduce LBW and PTD in countries such as PNG are rare; SPAZ is one such candidate worthy of further evaluation.

\section{Additional files}

Additional file 1: Study protocol.

Additional file 2: CONSORT checklist.

Additional file 3: Map of study area including location of recruitment antenatal clinics.

Additional file 4: Table S1. Comparison of baseline characteristics of study participants included in, and excluded from, the intention-to-treat (ITT) birthweight analysis. Table S2. Comparison of baseline characteristics of study participants excluded from the ITT birthweight analysis, by treatment arm $(n=754)$. Table S3. Factors associated with low birthweight on crude analysis. Table S4. Stratified analysis of low birthweight for gravidity, maternal height, maternal ethnic parentage, and bed net use before enrolment, by treatment group.

\section{Abbreviations}

AEs: Adverse events; ARR: Absolute risk reduction; AZ: Azithromycin; CQ: Chloroquine; DSMB: Data Safety Monitoring Board; GA: Gestational age; IPTp: Intermittent preventive treatment of malaria in pregnancy; ITT: Intention-to-treat; LBW: Low birthweight; P.f.: Plasmodium falciparum; PNG: Papua New Guinea; PNGIMR: PNG Institute of Medical Research; PP: Per-protocol; PTD: Pre-term delivery; P.v.: P. vivax; RR: Risk ratios; SAE: Serious adverse event; SPAZ: AZ combined with SP; SP:

Sulphadoxine-pyrimethamine; STIs: Sexually transmitted infections.

\section{Competing interests}

All authors declare that they have no competing interests.

\section{Authors' contributions}

SJR, IM, PS, CM, and AB conceived and designed the study. MO, RW, HWU, $\mathrm{SH}, \mathrm{DS}$, and IB supervised enrolment and follow-up of patients. AJU, LJR, $A R U, E L$, and CK supervised and conducted laboratory procedures. HWU, SJR, IM, CSNLWS, RW, MO, LJR, and DSu verified, analysed, and interpreted the data. HWU and SJR drafted the original version of the manuscript. All authors participated in the writing of the manuscript, and read and approved the final draft.

\section{Acknowledgements}

We thank the participating women and their families; the PNGIMR clinical, administrative, logistics, and laboratory staff; the staff at all participating health centres and hospitals; Lisa Lansfield, Hector Morris, John Bolnga, Jaume Ordi, Albert Serra Pou, Eline Kattenberg, Celine Barnadas, Andrew Raiko, Jane Walker, Sylvia Soso, Nola Ndrewei, Clare Ryan, and Jacob Kumai; DSMB members Julie Simpson and James McCarthy; independent clinician (drug safety) Moses Laman; independent clinical monitor Honor Rose; and Christalla Hajisava. This study was funded by the Malaria in Pregnancy Consortium, through a grant from the Bill \& Melinda Gates Foundation (46099); the Pregvax Consortium, through a grant from the European Union's Seventh Framework Programme FP7-2007-HEALTH (PREGVAX 201588) and the Spanish Government (EUROSALUD 2008 Programme); and Pfizer Inc., through an investigator-initiated research grant (WS394663). LJR received a National Health and Medical Research Council (NHMRC) Early Career 
Fellowship (\#1016443). IM received an NHMRC Senior Research Fellowship (\#1043345). The funders had no role in study design, data collection and analysis, decision to publish, or preparation of the manuscript.

\section{Author details}

'Department of Medicine (Royal Melbourne Hospital), The University of Melbourne, Post Office Royal Melbourne Hospital, Parkville, Victoria 3050, Australia. ${ }^{2}$ Papua New Guinea Institute of Medical Research, PO Box 60, Goroka, Eastern Highlands Province 441, Papua New Guinea. ${ }^{3}$ Walter and Eliza Hall Institute (WEHI), Parkville, Victoria 3052, Australia. ${ }^{4}$ Institute of Tropical Medicine, Nationalestraat 155, 2000 Antwerpen, Belgium. ${ }^{5}$ Barcelona Centre for International Health Research (CRESIB), Hospital Clínic-Universitat de Barcelona, Rossello, 132, 7th floor, 08036 Barcelona, Spain. ${ }^{6}$ Australian Institute of Tropical Health and Medicine, Faculty of Medicine, Health, and Molecular Sciences, James Cook University, Townsville, Queensland 4811, Australia. ${ }^{7}$ Department of Medical Biology, The University of Melbourne, Parkville, Victoria 3010, Australia.

\section{Received: 31 October 2014 Accepted: 16 December 2014} Published online: 16 January 2015

\section{References}

1. Guyatt HL, Snow RW. Impact of malaria during pregnancy on low birth weight in sub-Saharan Africa. Clin Microbiol Rev. 2004;17:760-9.

2. Umbers AJ, Aitken EH, Rogerson SJ. Malaria in pregnancy: small babies, big problem. Trends Parasitol. 2011;27:168-75.

3. Desai M, ter Kuile FO, Nosten F, McGready R, Asamoa K, Brabin B, et al. Epidemiology and burden of malaria in pregnancy. Lancet Infect Dis. 2007;7:93-104

4. Brabin BJ, Romagosa C, Abdelgalil S, Menendez C, Verhoeff FH, McGready R, et al. The sick placenta-the role of malaria. Placenta. 2004;25:359-78.

5. Aitken EH, Mbewe B, Luntamo M, Maleta K, Kulmala T, Friso MJ, et al. Antibodies to chondroitin sulfate A-binding infected erythrocytes: dynamics and protection during pregnancy in women receiving intermittent preventive treatment. J Infect Dis. 2010;201:1316-25.

6. Dellicour S, Tatem AJ, Guerra CA, Snow RW, ter Kuile FO. Quantifying the number of pregnancies at risk of malaria in 2007: a demographic study. PLoS Med. 2010;7:e1000221.

7. World Health Organization. WHO policy brief for the implementation of intermittent preventive treatment in malaria in pregnancy using sulfadoxine-pyrimethamine (IPTp-SP) (January 2014). Geneva: World Health Organization; 2014

8. Taylor SM, Antonia AL, Chaluluka E, Mwapasa V, Feng G, Molyneux ME, et al. Antenatal receipt of sulfadoxine-pyrimethamine does not exacerbate pregnancy-associated malaria despite the expansion of drug-resistant Plasmodium falciparum: clinical outcomes from the QuEERPAM study. Clin Infect Dis. 2012;55:42-50.

9. Chico RM, Chandramohan D. Intermittent preventive treatment of malaria in pregnancy: at the crossroads of public health policy. Trop Med Int Health. 2011;16:774-85.

10. Salman S, Rogerson SJ, Kose K, Griffin S, Gomorai S, Baiwog F, et al. Pharmacokinetic properties of azithromycin in pregnancy. Antimicrob Agents Chemother. 2010;54:360-6.

11. Luntamo M, Kulmala T, Mbewe B, Cheung YB, Maleta K, Ashorn P. Effect of repeated treatment of pregnant women with sulfadoxine-pyrimethamine and azithromycin on preterm delivery in Malawi: a randomized controlled trial. Am J Trop Med Hyg. 2010;83:1212-20.

12. Luntamo M, Rantala AM, Meshnick SR, Cheung YB, Kulmala T, Maleta K, et al. The effect of monthly sulfadoxine-pyrimethamine, alone or with azithromycin, on PCR-diagnosed malaria at delivery: a randomized controlled trial. PLoS One. 2012:7:e41123.

13. Chico RM, Hack BB, Newport MJ, Ngulube E, Chandramohan D. On the pathway to better birth outcomes? A systematic review of azithromycin and curable sexually transmitted infections. Expert Rev Anti Infect Ther. 2013;11:1303-32.

14. Sidhu AB, Sun $Q$, Nkrumah L, Dunne MW, Sacchettini JC, Fidock DA. In vitro efficacy, resistance selection, and structural modeling studies implicate the malarial parasite apicoplast as the target of azithromycin. J Biol Chem. 2007;282:2494-504.

15. Chandra RS, Orazem J, Ubben D, Duparc S, Robbins J, Vandenbroucke P. Creative solutions to extraordinary challenges in clinical trials: methodology of a phase III trial of azithromycin and chloroquine fixed-dose combination in pregnant women in Africa. Malar J. 2013;12:122.

16. Mueller I, Rogerson S, Mola GD, Reeder JC. A review of the current state of malaria among pregnant women in Papua New Guinea. P N G Med J. 2008;51:12-6.

17. Rijken MJ, McGready R, Boel ME, Poespoprodjo R, Singh N, Syafruddin D, et al. Malaria in pregnancy in the Asia-Pacific region. Lancet Infect Dis. 2012;12:75-88

18. Barnadas C, Senn N, Iga J, Timinao L, Javati S, Malau E, et al. Plasmodium falciparum and Plasmodium vivax genotypes and efficacy of intermittent preventive treatment in Papua New Guinea. Antimicrob Agents Chemother. 2014;58:6958-61.

19. Wong RP, Karunajeewa H, Mueller I, Siba P, Zimmerman PA, Davis TM. Molecular assessment of Plasmodium falciparum resistance to antimalarial drugs in Papua New Guinea using an extended ligase detection reaction fluorescent microsphere assay. Antimicrob Agents Chemother. 2010;55:798-805.

20. Wong RP, Lautu D, Tavul L, Hackett SL, Siba P, Karunajeewa HA, et al. In vitro sensitivity of Plasmodium falciparum to conventional and novel antimalarial drugs in Papua New Guinea. Trop Med Int Health. 2010;15:342-9.

21. Naidoo I, Roper C. Mapping 'partially resistant', 'fully resistant', and 'super resistant' malaria. Trends Parasitol. 2013;29:505-15.

22. Chico RM, Chandramohan D. Azithromycin plus chloroquine: combination therapy for protection against malaria and sexually transmitted infections in pregnancy. Expert Opin Drug Metab Toxicol. 2011;7:1153-67.

23. Schultz L, Wapling J, Mueller I, Ntsuke PO, Senn N, Nale J, et al. Multilocus haplotypes reveal variable levels of diversity and population structure of Plasmodium falciparum in Papua New Guinea, a region of intense perennial transmission. Malar J. 2010:9:336.

24. Vallely A, Page A, Dias S, Siba P, Lupiwa T, Law G, et al. The prevalence of sexually transmitted infections in Papua New Guinea: a systematic review and meta-analysis. PLoS One. 2010;5:e15586.

25. National Competency Training Project. Malaria diagnosis and the new treatment protocol: a training manual for health workers in Papua New Guinea. Port Moresby: Birdwing Publishing; 2010.

26. Loughna P, Chitty L, Evans T, Chudleigh T. Fetal size and dating: charts recommended for clinical obstetric practice. Ultrasound. 2009:17:161-7.

27. Hadlock FP, Deter RL, Harrist RB, Park SK. Estimating fetal age: computerassisted analysis of multiple fetal growth parameters. Radiology. 1984;152:497-501.

28. Rosanas-Urgell A, Mueller D, Betuela I, Barnadas C, Iga J, Zimmerman PA et al. Comparison of diagnostic methods for the detection and quantification of the four sympatric Plasmodium species in field samples from Papua New Guinea. Malar J. 2010;9:361.

29. Wangnapi RA, Soso S, Unger HW, Sawera C, Ome M, Umbers AJ, et al. Prevalence and risk factors for Chlamydia trachomatis, Neisseria gonorrhoeae and Trichomonas vaginalis infection in pregnant women in Papua New Guinea. Sex Transm Infect. 2014 [Epub ahead of print].

30. Ismail MR, Ordi J, Menendez C, Ventura PJ, Aponte JJ, Kahigwa E, et al. Placental pathology in malaria: a histological, immunohistochemical, and quantitative study. Hum Pathol. 2000;31:85-93.

31. Rogerson SJ, Pollina E, Getachew A, Tadesse E, Lema VM, Molyneux ME. Placental monocyte infiltrates in response to Plasmodium falciparum malaria infection and their association with adverse pregnancy outcomes. Am J Trop Med Hyg. 2003;68:115-9.

32. Rijken MJ, Rijken JA, Papageorghiou AT, Kennedy SH, Visser GH, Nosten F, et al. Malaria in pregnancy: the difficulties in measuring birthweight. BJOG. 2011;118:671-8.

33. Zou G. A modified Poisson regression approach to prospective studies with binary data. Am J Epidemiol. 2004;159:702-6.

34. Rubin DB. Multiple imputations for nonresponse in surveys. New York: Wiley \& Sons; 1987.

35. van den Broek NR, White SA, Goodall M, Ntonya C, Kayira E, Kafulafula G, et al. The APPLe study: a randomized, community-based, placebo-controlled trial of azithromycin for the prevention of preterm birth, with meta-analysis. PLOS Med. 2009;6:e1000191.

36. Kayentao K, Garner P, van Eijk AM, Naidoo I, Roper C, Mulokozi A, et al. Intermittent preventive therapy for malaria during pregnancy using 2 vs 3 or more doses of sulfadoxine-pyrimethamine and risk of low birth weight in Africa: systematic review and meta-analysis. JAMA. 2013;309:594-604.

37. Radeva-Petrova D, Kayentao K, Ter Kuile FO, Sinclair D, Garner P. Drugs for preventing malaria in pregnant women in endemic areas: any drug 
regimen versus placebo or no treatment. Cochrane Database Syst Rev. 2014;10, CD000169.

38. Zarogoulidis P, Papanas N, Kioumis I, Chatzaki E, Maltezos E, Zarogoulidis K. Macrolides: from in vitro anti-inflammatory and immunomodulatory properties to clinical practice in respiratory diseases. Eur J Clin Pharmacol. 2012;68:479-503

39. Harrington WE, Mutabingwa TK, Muehlenbachs A, Sorensen B, Bolla MC, Fried $\mathrm{M}$, et al. Competitive facilitation of drug-resistant Plasmodium falciparum malaria parasites in pregnant women who receive preventive treatment. Proc Natl Acad Sci U S A. 2009;106:9027-32.

40. Skalet AH, Cevallos V, Ayele B, Gebre T, Zhou Z, Jorgensen JH, et al. Antibiotic selection pressure and macrolide resistance in nasopharyngeal Streptococcus pneumoniae: a cluster-randomized clinical trial. PLoS Med. 2010;7:e1000377.

41. Lund M, Pasternak B, Davidsen RB, Feenstra B, Krogh C, Diaz L, et al. Use of macrolides in mother and child and risk of infantile hypertrophic pyloric stenosis: nationwide cohort study. BMJ. 2014;348:91908.

42. Brabin B, Piper C. Anaemia- and malaria-attributable low birthweight in two populations in Papua New Guinea. Ann Hum Biol. 1997;24:547-55.

43. National Department of Health. Standard management of sexually transmitted infections and genital conditions in Papua New Guinea. Port Moresby: National Department of Health; 2006.

44. Vallely LM, Homiehombo P, Kelly AM, Vallely A, Homer CS, Whittaker A. Exploring women's perspectives of access to care during pregnancy and childbirth: a qualitative study from rural Papua New Guinea. Midwifery. 2013;29:1222-9.

\section{Submit your next manuscript to BioMed Central and take full advantage of:}

- Convenient online submission

- Thorough peer review

- No space constraints or color figure charges

- Immediate publication on acceptance

- Inclusion in PubMed, CAS, Scopus and Google Scholar

- Research which is freely available for redistribution 